OPEN ACCESS

Edited by:

Cheng Guo,

Columbia University, United State

Reviewed by:

Shrikant Pawar.

Yale University, United States

Wen-Ming Cao,

University of Chinese Academy of

Sciences, China

*Correspondence:

Hui Yu

125391859@qq.com

Hongyan Wang

sunxiaodong1717@sina.com

${ }^{\dagger}$ These authors have contributed equally to this work

Specialty section:

This article was submitted to

Cancer Imaging and Image-directed Interventions,

a section of the journal

Frontiers in Oncology

Received: 20 January 2022 Accepted: 08 February 2022

Published: 03 March 2022

Citation:

Huang Z, Yang L, Chen J, Li S,

Huang J, Chen Y, Liu J, Wang $H$ and

Yu H (2022) CCDC134 as a

Prognostic-Related Biomarker

in Breast Cancer Correlating

With Immune Infiltrates.

Front. Oncol. 12:858487.

doi: 10.3389/fonc.2022.858487

\section{CCDC134 as a Prognostic-Related Biomarker in Breast Cancer Correlating With Immune Infiltrates}

\author{
Zhijian Huang ${ }^{1,2 \dagger}$, Linhui Yang ${ }^{1 \dagger}$, Jian Chen ${ }^{1}$, Shixiong $\mathrm{Li}^{1}$, Jing Huang ${ }^{3}$, Yijie Chen ${ }^{4}$, \\ Jingbo Liu $^{5}$, Hongyan Wang ${ }^{6 *}$ and Hui Yu ${ }^{3 *}$ \\ ${ }^{1}$ Department of Breast Surgical Oncology, Fujian Medical University Cancer Hospital, Fujian Cancer Hospital, Fuzhou, China, \\ 2 The Graduate School of Fujian Medical University, Fuzhou, China, ${ }^{3}$ Department of Pharmacy, Fujian Medical University Cancer \\ Hospital, Fujian Cancer Hospital, Fuzhou, China, ${ }^{4}$ Department of Ultrasound, Fujian Medical University Cancer Hospital, Fujian \\ Cancer Hospital, Fuzhou, China, ${ }^{5}$ Pathology Department, Daqing Longnan Hospital, The Fifth Affiliated Hospital of Qiqihar \\ Medical College, Daqing, China, ${ }^{6}$ Department of Pathology, Daqing Oiffield General Hospital, Daqing, China
}

Background: The expression of Coiled-Coil Domain Containing 134(CCDC134) is upregulated in different pan-cancer species. However, its prognostic value and correlation with immune infiltration in breast cancer are unclear. Therefore, we evaluated the prognostic role of CCDC134 in breast cancer and its correlation with immune invasion.

Methods: We downloaded the transcription profile of CCDC134 between breast cancer and normal tissues from the Cancer Genome Atlas (TCGA). CCDC134 protein expression was assessed by the Clinical Proteomic Cancer Analysis Consortium (CPTAC) and the Human Protein Atlas. Gene set enrichment analysis (GSEA) was also used for pathway analysis. Receiver operating characteristic $(\mathrm{ROC})$ curve was used to differentiate breast cancer from adjacent normal tissues. Kaplan-Meier method was used to evaluate the effect of CCDC134 on survival rate. The protein-protein interaction (PPI) network is built from STRING. Function expansion analysis is performed using the ClusterProfiler package. Through tumor Immune Estimation Resource (TIMER) and tumor Immune System Interaction database (TISIDB) to determine the relationship between CCDC134 expression level and immune infiltration. CTD database is used to predict drugs that inhibit CCDC134 and PubChem database is used to determine the molecular structure of identified drugs.

Results: The expression of CCDC134 in breast cancer tissues was significantly higher than that of CCDC134 mRNA expression in adjacent normal tissues. ROC curve analysis showed that the AUC value of CCDC134 was 0.663. Kaplan-meier survival analysis showed that patients with high CCDC134 had a lower prognosis (57.27 months vs 36.96 months, $\mathrm{P}=2.0 \mathrm{E}-6)$. Correlation analysis showed that CCDC134 mRNA expression was associated with tumor purity immune invasion. In addition, CTD database analysis identified abrine, Benzo (A) Pyrene, bisphenol A, Soman, Sunitinib, Tetrachloroethylene, 
Valproic Acid as seven targeted therapy drugs that may be effective treatments for seven targeted therapeutics. It may be an effective treatment for inhibiting CCDC134.

Conclusion: In breast cancer, upregulated CCDC134 is significantly associated with lower survival and immune infiltrates invasion. Our study suggests that CCDC134 can serve as a biomarker of poor prognosis and a potential immunotherapy target in breast cancer. Seven drugs with significant potential to inhibit CCDC134 were identified.

Keywords: breast cancer, CCDC134, biomarker, prognosis, immune infiltration

\section{INTRODUCTION}

Breast cancer (BC) has overtaken lung cancer as the most common cancer worldwide and is the most common cancer in women (1). Over the past 20 years, the number of new cases of breast cancer has gradually increased globally, from 1.15 million (2002) (2) to 1.38 million (2008) (3), 1.68 million (2012) (4) and 2.09 million (2018) (5); The projected figure for 2050 is about 3.2 million (6). Thanks to advances in early screening and the development of anti-cancer strategies, the treatment of breast cancer has improved significantly. However, the recurrence rate remains high (7-9). Studies have shown that the prognosis of breast cancer is influenced by a variety of clinical factors (10), such as age, tumor size, histological grade, lymphatic infiltration, number of lymph node metastases, hormone receptor status, Her-2 status, and positive margins. Due to the complexity of the onset of breast cancer and the heterogeneity of tumors, although many prognostic markers have been found, the prediction efficiency is still inadequate $(11,12)$. It is necessary to build a new breast cancer risk prediction model to improve the treatment and prognosis of breast cancer patients.

Relevant studies have reported that $\mathrm{CCDCl} 34$ is a newly discovered secreted protein screened by Huang et al. (13) through cell chip in 2008, which is widely expressed in a variety of human normal tissues, cancer tissues and cell lines. CCDC134 is a protein coding gene. Disorders associated with $C C D C 134$ include Ehlers-Danlos syndrome, hyperactivity type. CCDC134 is reported to be a novel CD8+T cell stimulator that promotes proliferation and activation of $\mathrm{CD} 8+\mathrm{T}$ cells in exocrine form, suggesting a cytokine like function (14). It has been reported that this gene may affect ERK and JNK signaling activity in gastric cancer cells $(13,15)$.

At present, the relationship between $\mathrm{CCDCl} 34$ and breast tumors has not been reported. We hypothesized that CCDC134 levels were associated with breast cancer survival. To test this hypothesis, we evaluated the prognostic role of CCDC134 in breast Cancer based on data from the Cancer Genome Atlas (TCGA). In this study, we found that CCDC134 is up-regulated in breast cancer. Notably, upregulation of CCDC134 was associated with adverse clinical features and risk factors. We further evaluated the diagnostic and prognostic value of CCDC134 in breast cancer and its correlation. Our study suggests that overexpression of CCDC134 in breast cancer is associated with lower survival.

\section{MATERIALS AND METHODS}

\section{TCGA Datasets}

Download transcription and expression data of CCDC134 and corresponding clinical information from TCGA official website (16). More than 5 samples of the 33 registered cancers were selected for analysis. Finally, the RNA-SEQ gene expression data of workflow type FPKM were transformed into TPM format and $\log 2$ transformation for further study. As all data were downloaded from TCGA, no approval from the Ethics Committee was required for this study. We provided the database links for this article in Supplementary Table 1.

\section{RNA Sequencing Data of CCDC134 in Breast Cancer}

RNA-seq expression data of CCDC134 in breast cancer were also downloaded from TCGA and the XIANTAO platform (https:// www.xiantao.love/). Therefore, data on 1109 breast cancers and 113 adjacent normal tissues were retained. The selected samples contained CCDC134 gene expression data and relevant clinical information, including age, sex, smoking status, $\mathrm{T}$ stage, $\mathrm{N}$ stage, $M$ stage, tumor site, ER/PR/HER2 status, etc. The mRNA expression data were the mean of $\mathrm{X} \pm \mathrm{SD}$.

\section{Clinical Proteomic Tumor Analysis Consortium (CPTAC) and UALCAN}

CPTAC (https://proteomics.cancer.gov/programs/cptac) application of proteomics techniques, by mass spectrometry analysis of tumor biological specimens, quantification and identification of each tumor sample, the composition of the protein and the protein group were characterized (17). UALCAN [http://ualcan.path.uab.edu/] is a user-friendly online resource for analyzing publicly available cancer data (18). In this study, we used UALCAN to analyze the expression of CCDC134 protein in CPTAC.

\section{The Human Protein Atlas (HPA)}

HPA (https://proteinatlas.org/) contains normal tissue and tumor tissue protein levels of human gene expression profile information (19). In this study, we compared the expression of CCDC134 protein in normal lung tissue and breast cancer tissue by HPA. 


\section{GSEA (Gene Set Enrichment Analysis) Functional and Pathway Analysis}

GSEA (20) was performed using clusterProfiler, enrichPlot and ggplot2 $\mathrm{R}$ packet (V 3.3.3) to demonstrate important functions and pathways between the two groups. The expression level of CCDC134 was used as a phenotypic marker. Adjusted p values $<0.05$, the enrichment of standardized scores $(|\mathrm{NES}|)<1$, the false discovery rate (FDR) $<0.25$ is significant difference.

\section{Construction and Evaluation of Line Graph}

Individual prediction of 1-year, 3-year and 5-year survival probability (21). Based on the results of multiple variable analysis, the column chart was constructed. The RMS R package (version 6.2-0) is used to generate a Nomogram with significant clinical features and calibration diagrams. C concordiindex and correction curve were used to estimate its predictive ability.

\section{Protein-Protein Interaction (PPI) Networks and Functional Enrichment Analysis}

STRING is an online database for retrieving interacting genes [version 11.0 (https://www.string-db.org/)] (22). In this study, we used STRING to search for co-expressed genes and construct a PPI network $(23,24)$, with an interaction score of 0.4 . Analysis of Kyoto Encyclopedia of Genes and Genomes (KEGG) pathways enriched by gene ontology (GO) and co-expressed genes was performed using ClusterProfiler software package and GGploT2 software package visualization (20).

\section{Tumor Immune Estimation Resource (TIMER) Database}

The TIMER (https://cistrome.shinyapps.io/timer/) is a comprehensive online database, analysis of a wide variety of cancer types related to immune infiltrating (25). In this study, we used TIMER to determine the relationship between CCDC134 expression and six types of immune infiltrates (B cells, CD4+ T cells, CD8+ T cells, neutrophils, macrophages, and dendritic cells) in breast cancer.

\section{Tumor-Immune System Interaction Database (TISIDB)}

TISIDB (http://cis.hku.hk/TISIDB/) is a tumor-immune system interaction online portal (26). In this study, we used TISIDB to determine CCDC134 and tumor-infiltrating lymphocyte (TILs) expression in human cancers. The relative abundance of TILs was deduced from gene expression profile and gene set variation analysis. Spearman test was used to measure the correlation between CCDC134 and TILs. The relative abundance threshold was set as $|\mathrm{R}|>0.5$, P-value $<0.05$.

\section{Recurrence - Free Survival (RFS) Data Analysis}

Survival data from KMPLOT online analysis database (https:// kmplot.com/analysis/index.php?p = service). Affy IDS of
CCDC134 are 220077_at. "Auto select best truncation value" and "Basic type" were selected for breast cancer analysis.

\section{Screening of Small Molecule Therapeutic Drugs}

The selected genes are used for potential drug prediction in CTDBase. CTD database (https://ctdbase.org/). can be used to advance the understanding of chemical medicines and human health based on studies of the relationships between chemistry, genes, phenotypes, diseases and the environment. PubChem database (https://pubchem.ncbi.nlm.nih.gov/) is used to determine the identified molecular structure of the drug.

\section{Statistical Analyses}

All statistical analyses were performed using R (V 3.6.3), and differences were visualized using $\mathrm{R}$ package GGplot2 (20), clusterProfiler package [version 3.14.3] (for GSEA analysis). Paired $T$ test and Mann-Whitney $U$ test were used to determine the difference between breast cancer tissue and adjacent normal tissue. ROC curves were used to detect CCDC134 cutoff values (27-29) using pROC packages.

\section{RESULTS}

\section{Expression Pattern of CCDC134 in Pan-Cancer Perspective}

To assess the mRNA expression pattern of CCDC134 in different cancer types, 15 cancer types with fewer than 5 samples from the normal group were excluded from the analysis. The final working set involved 18 cancer types. As shown in Figure 1, CCDC134 was significantly upregulated in 14 of the 18 cancers compared with normal tissue. This data suggests that CCDC134 mRNA expression is abnormal in different cancer types.

\section{Upregulated mRNA and Protein Expression of CCDC134 in Patients With Breast Cancer}

To detect CCDC134 mRNA and protein expression in breast cancer, we analyzed CCDC134 expression data in TCGA and HPA.As shown in Figure 2A, unpaired data analysis showed that CCDC134 mRNA expression level in breast cancer tissues $(\mathrm{n}=$ 1109) was significantly higher than that in normal tissues $(\mathrm{n}=$ 113)(Figure 2A, 3.391 \pm 0.636 vs 3.005 \pm 0.618 , The Mann Whitney $\mathrm{U}$ - test, $\mathrm{P}<0.001)$. Paired data analysis also showed that mrna expression level of CCDC134 in breast cancer tissues ( $\mathrm{n}=112$ ) was significantly higher than that in adjacent normal tissues ( $\mathrm{n}=112$ ) (Figure 2B, 3.351 \pm 0.597 vs $2.998 \pm 0.617$, $\mathrm{P}<0.001)$. In order to comprehensively analyze CCDC134 protein expression, we used UALCAN to analyze CPTAC. The results showed that CCDC134 protein expression in breast cancer was significantly higher than that in normal tissues (Figure 2C). As shown in Figures 2D, E, HPA immunohistochemical staining also showed up-regulation of CCDC134 protein expression in breast cancer tissues. These results showed that both mRNA and 


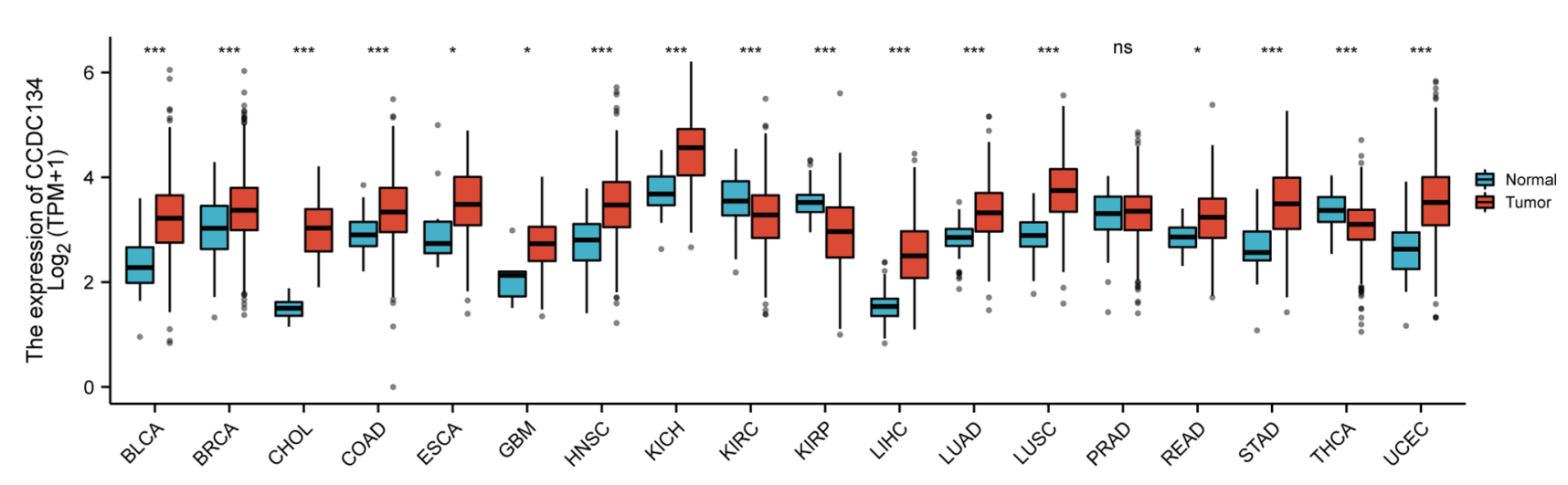

FIGURE 1 | Expression pattern of CCDC134 from the perspective of pan-cancer. CCDC134 mRNA expression was significant in 17 of the 18 cancers compared with normal tissue. (ns, $\mathrm{p} \geq 0.05$; ${ }^{\star} \mathrm{p}<0.05 ;{ }^{\star \star *} \mathrm{p}<0.001$ ). BLCA, Bladder Urothelial Carcinoma; BRCA, Bladder Urothelial Carcinoma; CHOL, Cholangiocarcinoma, COAD, Colon adenocarcinoma; ESCA, Esophageal carcinoma, GBM, Glioblastoma multiforme; HNSC, Head and Neck squamous cell carcinoma; KICH, Kidney Chromophobe; KIRC, Kidney renal clear cell carcinoma; KIRP, Kidney renal papillary cell carcinoma; LIHC, Liver hepatocellular carcinoma; LUAD, Lung adenocarcinoma; LUSC, Lung squamous cell carcinoma; PRAD, Prostate adenocarcinoma; READ, Rectum adenocarcinoma; STAD, Stomach adenocarcinoma; THCA, Thyroid carcinoma; UCEC, Uterine Corpus Endometrial Carcinoma.

protein expression of CCDC134 were up-regulated in breast cancer tissues.

\section{Gene Set Enrichment Analysis (GSEA)}

In order to understand the biological function of CCDC134, we analyzed the DEGs between the low and high CCDC134 expression groups according to the median expression value of CCDC134. GSEA pathway analysis was also performed (Supplementary Table 2) (Figure 3). The result shows that CCDC134 is enriched in CELL_CYCLE, REACTOME_DNA_ REPLICATION, and WP_PI3KAKTMTOR_VITD3. Low tolerance_by_vasoactive_INte and REACTOME_FCERI_ MEDIATED_NF_KB_ACTIVATION were found (Figure 3). At the same time, we completed the GO analysis(Supplementary Table 3). BP and keratinization, differentiation, chemical stimulation involved in detecting bitter sensory perception, epidermal cell differentiation. The first five CC terms are associated with cajal bodies, nucleosomes, DNA packaging complexes, keratin filaments, and intermediate filaments. The first five MF terms were associated with bitter taste receptor activity, channel activity, passive transmembrane transporter activity, inhibitory extracellular ligand gated ion channel activity, and taste receptor activity (Figure 3B).

\section{Clinicopathological Features}

To evaluate the relationship between CCDC134 mRNA expression and clinicopathological features in breast cancer samples, mann-Whitney $U$ test and Logistic regression analysis were performed. As shown in Table 1 and Figure 4, CCDC134 expression was observed to have a strong association with $\mathrm{PR}$ status $(\mathrm{P}=0.002)$, ER status $(\mathrm{P}<0.001)$ and PAM50 types $(\mathrm{P}<$ 0.001). However, CCDC134 expression was not associated with T stage $(P=0.640), \mathrm{N}$ stage $(\mathrm{P}=0.545), \mathrm{M}$ stage $(\mathrm{P}=0.921)$, age $(\mathrm{P}=1740.081)$, pathological stage $(\mathrm{P}=0.786)$ and HER2 status $(\mathrm{P}=0.737)$. In summary, these results suggest that CCDC134 was involved in hormone receptor levels. Since the efficacy and prognosis of endocrine therapy for breast cancer were closely related to hormone receptor expression level, the results indicated that CCDC134 might be a biomarker of efficacy and prognosis of BC.

\section{Diagnostic and Prognostic Value}

In order to study the diagnostic value of CCDC134 in distinguishing breast cancer samples from normal breast cancer, ROC curve analysis was conducted. As shown in Figure 5A, ROC curve analysis shows that the AUC value of CCDC134 is $0.663,95 \%$ Confidence interval $(95 \% \mathrm{CI})=0.611$ 0.715 . When the critical value was 2.904 , the sensitivity was 0.460 and the specificity was 0.800 . The positive predictive value was 0.190 , and the negative predictive value was 0.936 . The results suggest that CCDC134 may be a promising biomarker for differentiating adenocarcinoma tissue from normal tissue. The relationship between CCDC134 mRNA expression and RFS in breast cancer patients was explored by Kaplan-Meier curve. As shown in Figure 5B, the RFS of patients with high level CCDC134 breast cancer was shorter than that of patients with low level CCDC134 breast cancer (57.27 months vs. 36.96 months, $\mathrm{P}=2.0 \mathrm{E}-6)$.

\section{Constructed a Nomogram Diagram}

To provide a quantitative method for predicting the prognosis of BRCA patients, we constructed a Nomogram diagram of CCDC134 and independent clinical risk factors (T/N/M stage, CCDC134, age, PAM50). In this Nomogram based on multivariate Cox analysis, a point scale is used to assign points to these variables. Draw a straight line up to determine the number of points for a variable and adjust the sum of points assigned to each variable to a range from 0 to 100 . The integrals of the variables are added up and recorded as an overall score. 
A

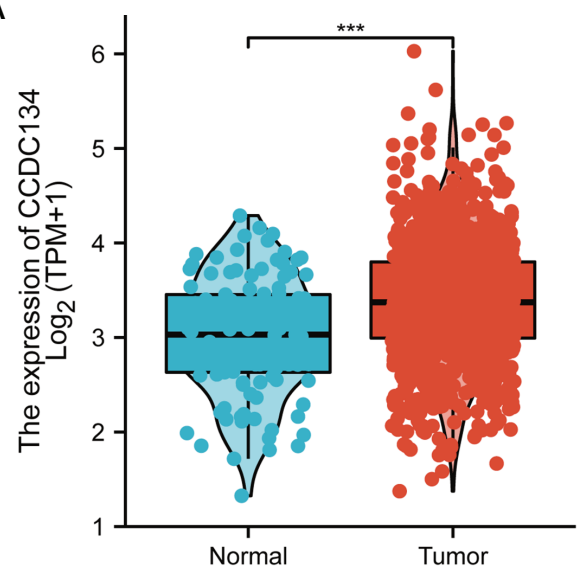

B

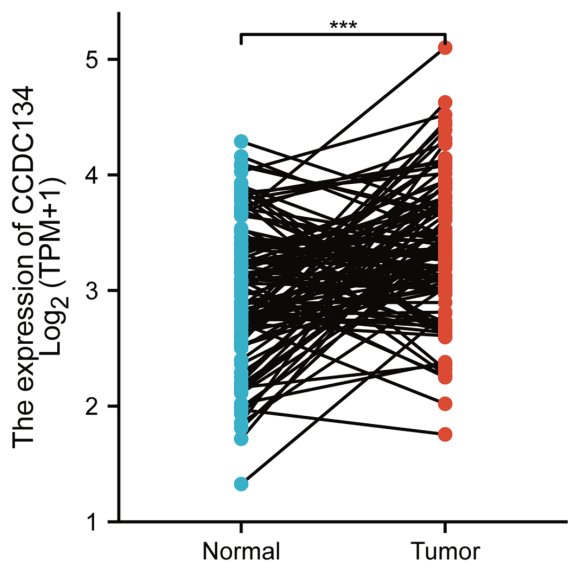

C

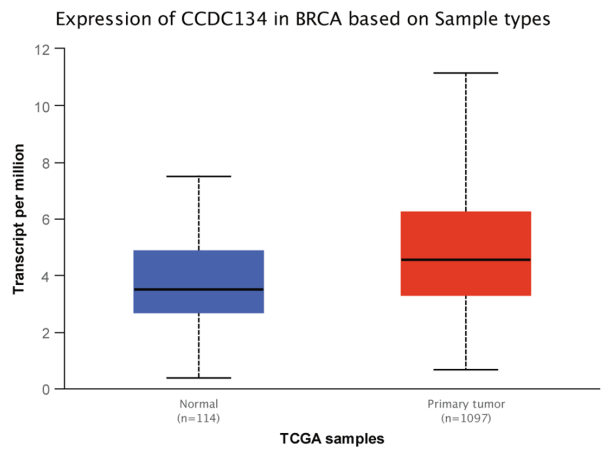

D

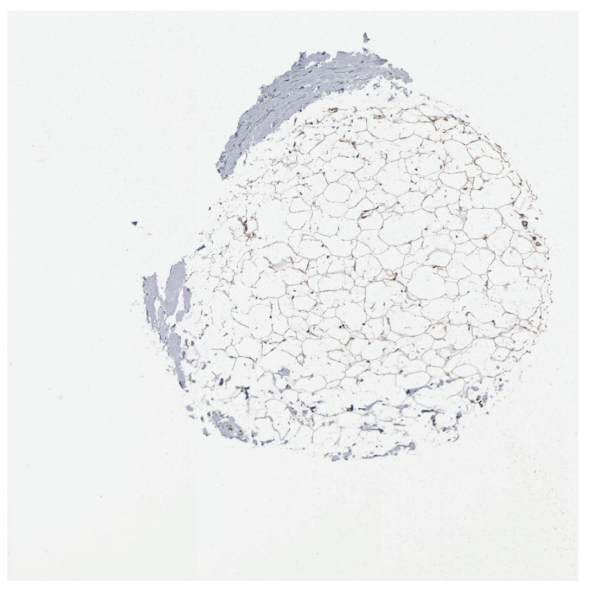

E

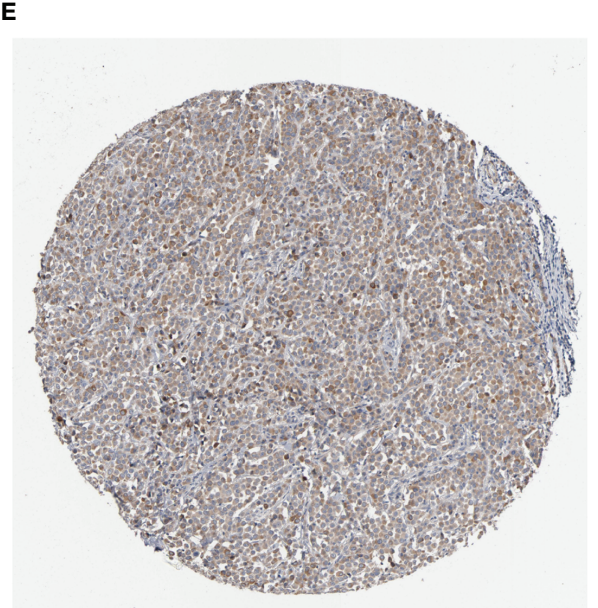

FIGURE 2 | CCDC134 mRNA and protein expression in breast cancer. (A) CCDC134 mRNA expression levels in 1109 breast and 113 normal breast cancers. (B) CCDC134 mRNA expression levels in 112 breast cancer patients and matched adjacent normal samples. (C) CCDC134 protein expression level based on CPTAC. (D) CCDC134 protein levels based on Human Protein Atlas. Normal tissue, https://www.proteinatlas.org/ENSG00000100147-CCDC134/tissue/breast\#img; (E) Tumor tissue, https://www.proteinatlas.org/ENSG00000100147-CCDC134/pathology//breast+cancer\#img. $\left(^{\star \star \star}\right.$ p $\left.<0.001\right)$.

The 1-year, 3-year, and 5-year survival probabilities for BRCA patients were determined vertically from the total point axis down to the outcome axis (Figure 6A). The nomogram C-index of OS is predicted to be $0.729(0.705-0.753)$ (Figure 6B).

\section{PPI Network and Functional Annotation}

To build the PPI network and functional annotations, we performed STRING database, GO and KEGG analysis. Figure 7A showed the network of CCDC134 and its 10 co- 
A

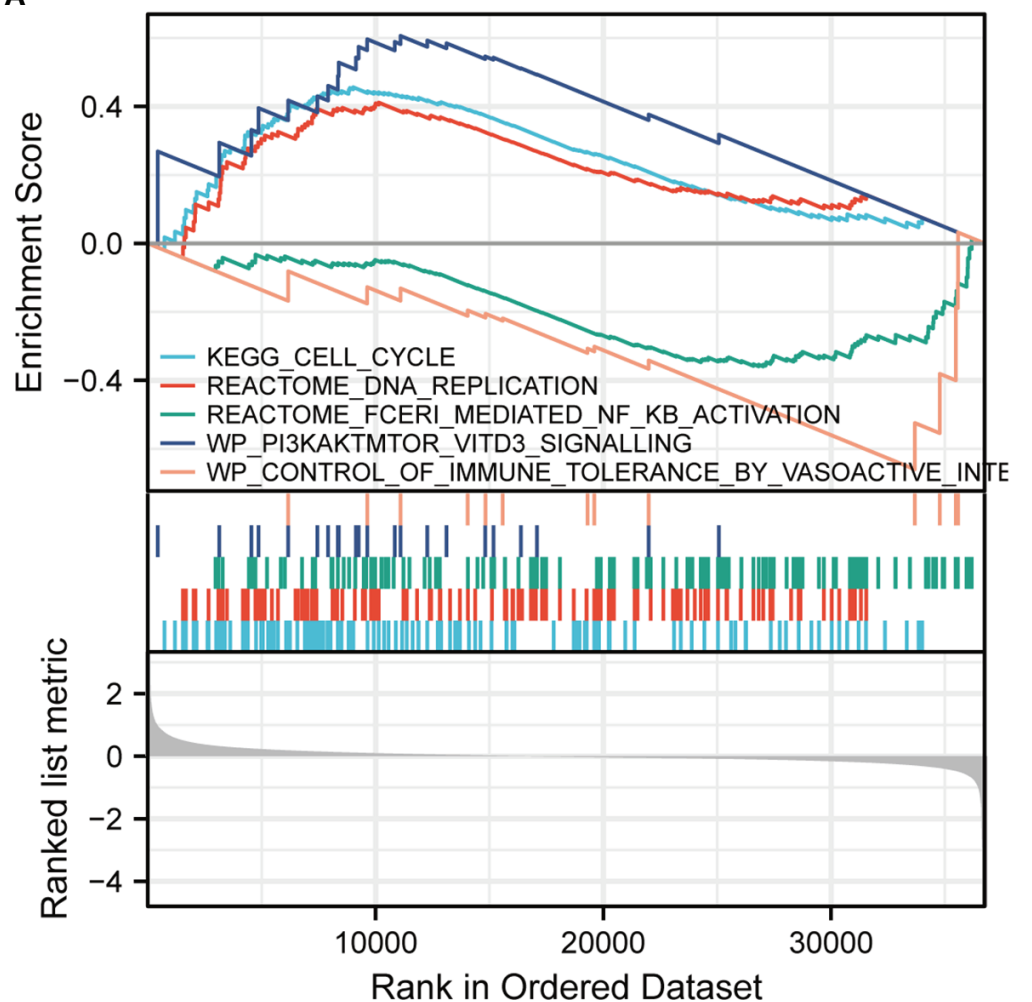

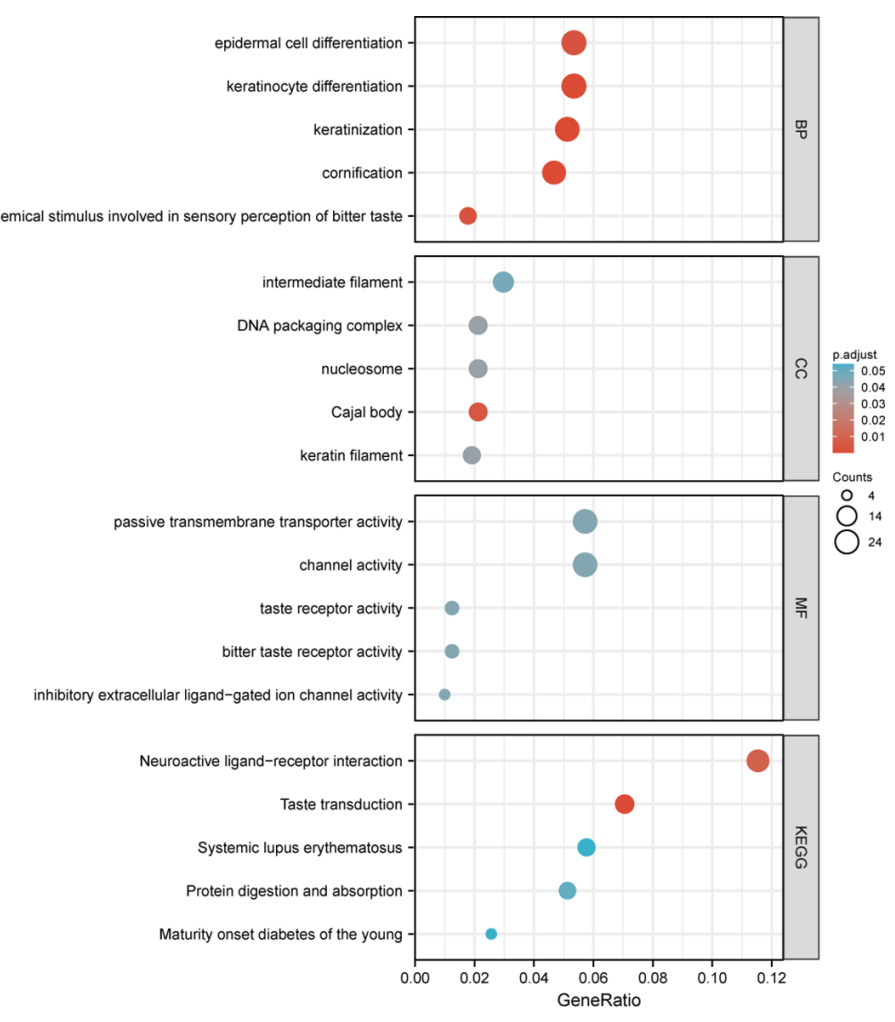

FIGURE 3 | GSEA analysis results. (A) Genes enriched in representative pathways were analyzed by GSEA function. (B) GO and KEGG analysis of DEGs in low and high expression samples of CCDC134. 
TABLE 1 | Clinical characteristics of the Breast invasive carcinoma patients (TCGA).

\begin{tabular}{|c|c|c|c|}
\hline Characteristic & $\begin{array}{l}\text { Low expression } \\
\text { of CCDC134 }\end{array}$ & $\begin{array}{l}\text { High expression } \\
\text { of CCDC134 }\end{array}$ & $\mathbf{p}$ \\
\hline$n$ & 541 & 542 & \\
\hline T stage, n (\%) & & & 0.640 \\
\hline $\mathrm{T} 1$ & 137 (12.7\%) & $140(13 \%)$ & \\
\hline T2 & $318(29.4 \%)$ & $311(28.8 \%)$ & \\
\hline T3 & 72 (6.7\%) & 67 (6.2\%) & \\
\hline $\mathrm{T} 4$ & $14(1.3 \%)$ & $21(1.9 \%)$ & \\
\hline N stage, n (\%) & & & 0.545 \\
\hline NO & $248(23.3 \%)$ & 266 (25\%) & \\
\hline N1 & 190 (17.9\%) & $168(15.8 \%)$ & \\
\hline N2 & $58(5.5 \%)$ & $58(5.5 \%)$ & \\
\hline N3 & $40(3.8 \%)$ & $36(3.4 \%)$ & \\
\hline M stage, n (\%) & & & 0.516 \\
\hline MO & $450(48.8 \%)$ & $452(49 \%)$ & \\
\hline M1 & 8 (0.9\%) & $12(1.3 \%)$ & \\
\hline Age, n (\%) & & & 0.081 \\
\hline$<=60$ & $315(29.1 \%)$ & 286 (26.4\%) & \\
\hline$>60$ & 226 (20.9\%) & 256 (23.6\%) & \\
\hline Pathologic stage, n (\%) & & & 0.786 \\
\hline Stage I & 90 (8.5\%) & 91 (8.6\%) & \\
\hline Stage II & 315 (29.7\%) & 304 (28.7\%) & \\
\hline Stage III & $123(11.6 \%)$ & $119(11.2 \%)$ & \\
\hline Stage IV & 7 (0.7\%) & $11(1 \%)$ & \\
\hline Histological type, n (\%) & & & 0.001 \\
\hline Infiltrating Ductal Carcinoma & $373(38.2 \%)$ & 399 (40.8\%) & \\
\hline Infiltrating Lobular Carcinoma & 126 (12.9\%) & 79 (8.1\%) & \\
\hline PR status, $n(\%)$ & & & 0.002 \\
\hline Negative & 145 (14\%) & 197 (19.1\%) & \\
\hline Indeterminate & $3(0.3 \%)$ & $1(0.1 \%)$ & \\
\hline Positive & $365(35.3 \%)$ & $323(31.2 \%)$ & \\
\hline ER status, n (\%) & & & $<0.001$ \\
\hline Negative & $91(8.8 \%)$ & 149 (14.4\%) & \\
\hline Indeterminate & $1(0.1 \%)$ & $1(0.1 \%)$ & \\
\hline Positive & $421(40.7 \%)$ & 372 (35.9\%) & \\
\hline HER2 status, n (\%) & & & 0.737 \\
\hline Negative & 279 (38.4\%) & $279(38.4 \%)$ & \\
\hline Indeterminate & $7(1 \%)$ & 5 (0.7\%) & \\
\hline Positive & 75 (10.3\%) & $82(11.3 \%)$ & \\
\hline PAM50, n (\%) & & & $<0.001$ \\
\hline Normal & $22(2 \%)$ & $18(1.7 \%)$ & \\
\hline LumA & $310(28.6 \%)$ & 252 (23.3\%) & \\
\hline LumB & $103(9.5 \%)$ & $101(9.3 \%)$ & \\
\hline Her2 & $35(3.2 \%)$ & 47 (4.3\%) & \\
\hline Basal & $71(6.6 \%)$ & $124(11.4 \%)$ & \\
\hline
\end{tabular}

expressed genes (Supplementary Table 4). As shown in Figure 7B, changes in CCDC134 bioprocesses were related to sphingomyelin metabolism, active regulation of glycolysis, positive regulation of nucleotide catabolism, active regulation of coenzyme metabolism, and assembly of ribosome macrosubunits. Functional annotations showed that these genes were involved in 5S rRNA binding, phosphotransferase activity for other substituted phosphate groups, rRNA binding, histone acetyl transferase activity, peptide-lysine- $\mathrm{N}$-acetyl transferase activity and other functions. The correlation analysis of CCDC134 expression and co-expressed genes in TCGA breast cancer was shown in Figures 7C-J (successfully converted to 9 Entrez ID).

\section{Correlation Between CCDC134 and Immune Cell Infiltration}

We analyzed the correlation between CCDC134 expression and six types of tumor-infiltrating immune cells in the TIMER database. As shown in Figure 8A, CCDC134 expression was correlated with tumor purity $(\mathrm{r}=0.023, \mathrm{P}=4.68 \mathrm{E}-01)$, B cells $(\mathrm{R}=0.125, \mathrm{P}=9.54 \mathrm{E}-05), \mathrm{CD} 8+\mathrm{T}$ cells $(\mathrm{r}=0.108, \mathrm{P}=7.38 \mathrm{E}$ $-04), \mathrm{CD} 4+\mathrm{T}$ cells $(\mathrm{r}=0.101,1.66 \mathrm{E}-03)$, macrophages $(\mathrm{r}=$ $0.101, \mathrm{P}=1.54 \mathrm{E}-03)$, neutrophils $(\mathrm{r}=0.189, \mathrm{P}=4.62 \mathrm{E}-09)$ and dendritic cells $(\mathrm{r}=0.165, \mathrm{P}=2.92 \mathrm{E}-07)$ were correlated. Figure 8B shows the correlation between CCDC134 and PDL1 (CD274) ( $\mathrm{r}=0.214, \mathrm{P}=7.89 \mathrm{E}-13)$. We also assessed the correlation between CCDC134 expression and 28 types of lymphocytes in the TISIDB database. Figure $\mathbf{8 C}$ shows the relationship between CCDC134 expression and 28 types of lymphocytes in human cancers. As shown in Figures 8D-K, the expression of CCDC134 was correlated with that of CCDC134 and Act_CD4 $+\mathrm{T}$ cells $(\mathrm{r}=0.105, \mathrm{P}=0.000486)$, DC cells $(\mathrm{r}=0.192, \mathrm{P}=1.61 \mathrm{E}-10)$, MDSC cells $(\mathrm{r}=0.073, \mathrm{P}=$ $0.0153)$, monocytes $(r=0.063, P=0.0375)$, pDC cells $(r=0.074$, $\mathrm{P}=0.0144)$, Tgd cells $(\mathrm{r}=0.083, \mathrm{P}=0.00578)$ and the abundance of Tcm_CD4 + T cells ( $\mathrm{r}=0.091, \mathrm{P}=0.00246)$ and CD56DIM ( $\mathrm{r}=$ $0.072, P=0.0173)$. These data suggest that $C C D C 134$ may play a specific role in immune invasion of breast cancer.

\section{Small Molecule Therapeutics}

The correlation between CCDC134 and potential drugs was analyzed using CTD database(Supplementary Table 5). A total of seven drugs were identified, including abrine, Benzo(A) Pyrene, bisphenol A, Soman, Sunitinib, Tetrachloroethylene and Valproic Acid (Figure 9). These drugs have a potential inhibitory effect on CCDC134.

\section{DISCUSSION}

In this study, we found that CCDC134 mRNA and protein expression were up-regulated in breast cancer tissues. ROC curve analysis indicated that CCDC134 may be a promising diagnostic biomarker for differentiating breast cancer from normal tissues. Using Kaplan-Meier curves and univariate analysis, we confirmed that CCDC134 expression is associated with short RFS and that CCDC134 can serve as a potential biomarker of poor prognosis in breast cancer. In addition, CCDC134 may play a specific role in immune invasion of breast cancer.

Studies have shown (30) that CCDC134 is a novel gene involved in severe progressive deformation recessive osteogenesis imperfecta (type III). (15)Decreased CCDC134 expression has been reported to enhance erK1/2 activation and JNK/SAPK expression. CCDC134 regulates cell migration and invasion and may be a therapeutic target for gastric cancer. Exposure to CCDC134 promotes proliferation of $\mathrm{CD} 8+\mathrm{T}$ cells through the Janus kinase 3- signal transductor and transcriptional activator 5 pathway. Two members of the $\gamma \mathrm{c}$ cytokine family effectively block CCDC134 binding to activated 
A

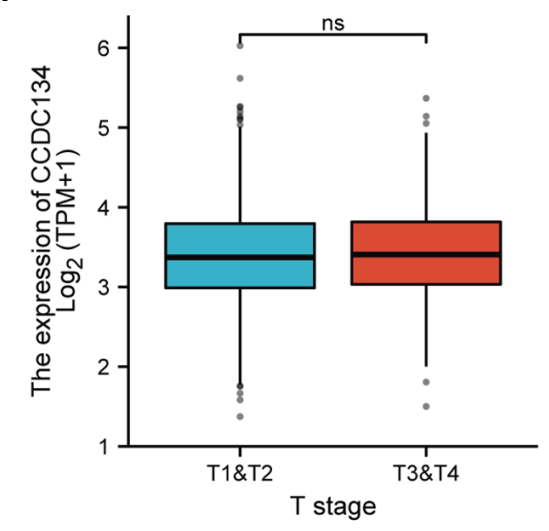

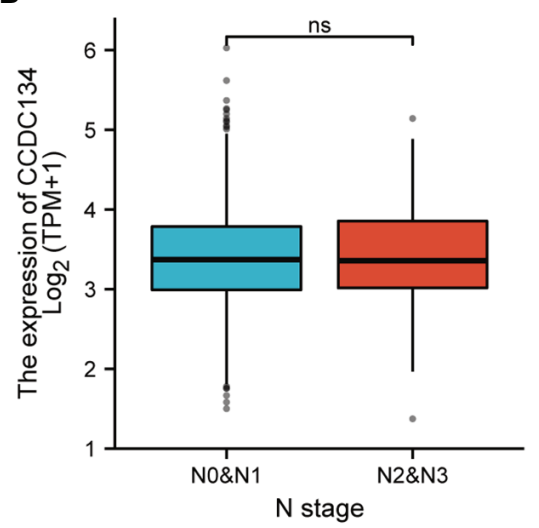

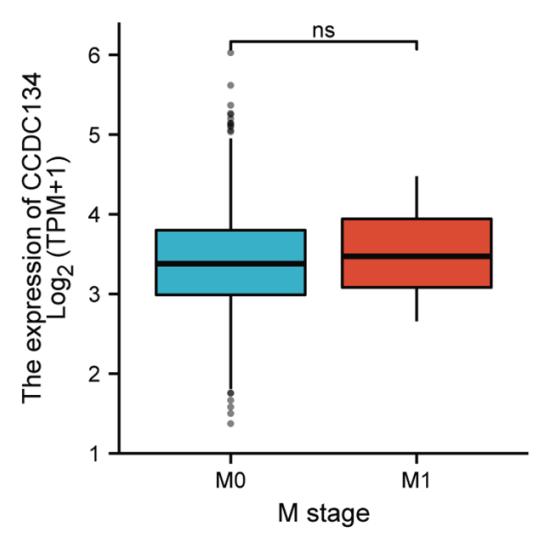

D

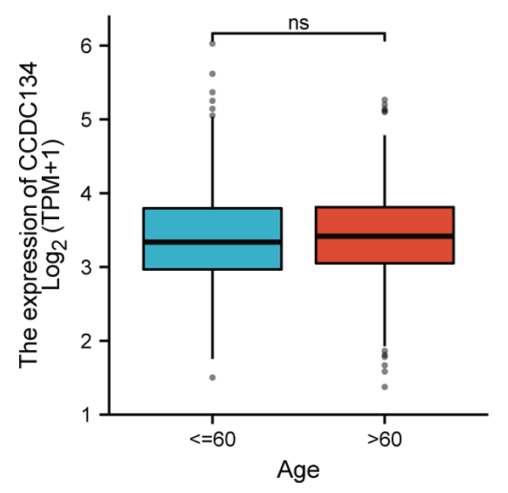

E

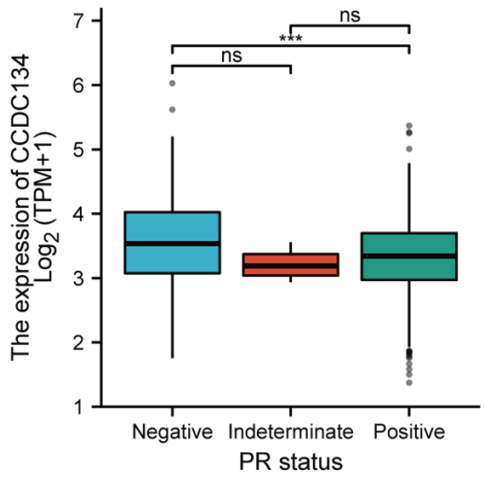

$\mathbf{F}$

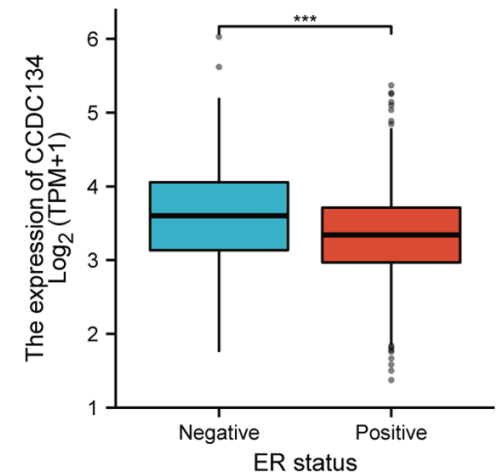

G

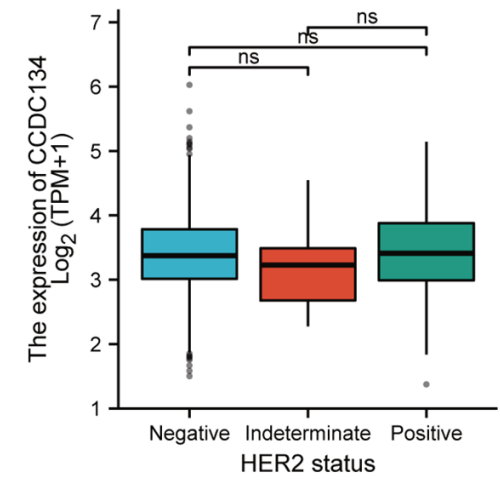

FIGURE 4 | CCDC134 mRNA level relationship with clinical pathological characteristics. There was no significant difference between CCDC134 mRNA expression and T (A), N (B), M (C) level, age (D) and HER2

(G) level. It was negatively correlated with PR (E) and ER (F) status. (ns, $p \geq 0.05$; $\left.{ }^{\star \star \star} p<0.001\right)$. 


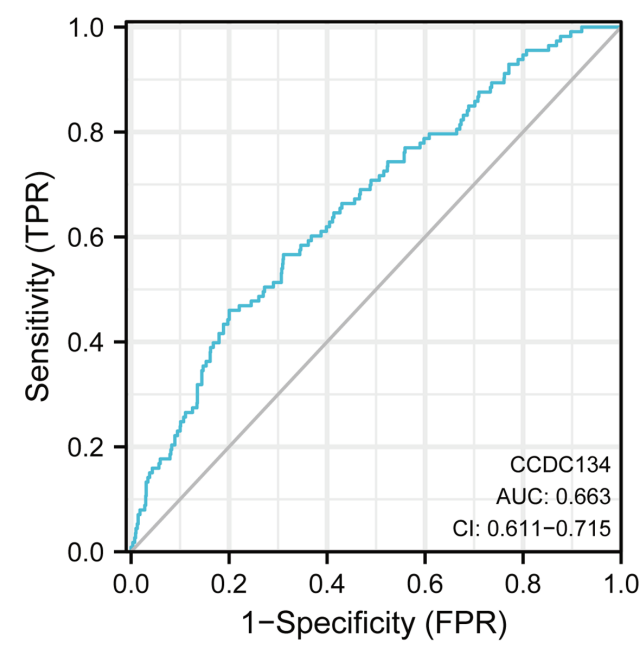

B

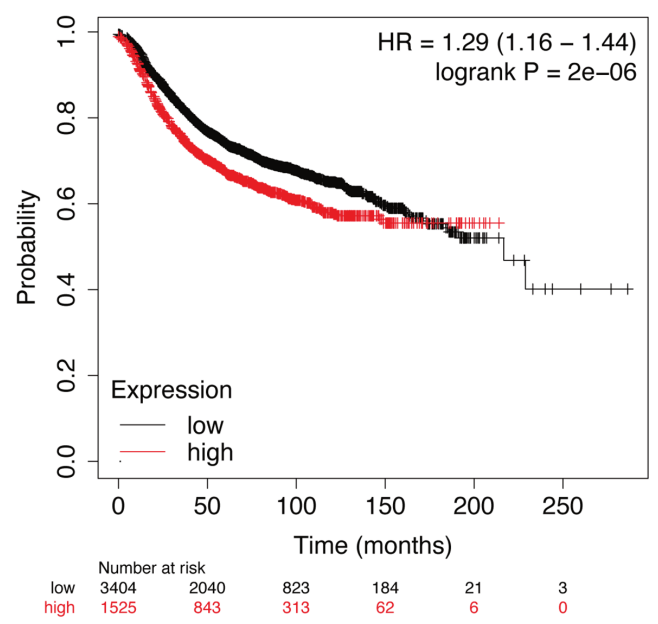

FIGURE 5 | ROC and Kaplan-Meier curves of CCDC134. (A) THE ROC curve showed that the AUC value of CCDC134 was 0.663 . (B) Kaplan-Meier survival curve showed that the RFS of breast cancer patients with high CCDC134 mRNA expression was shorter (57.27 months vs. 36.96 months) than that of breast cancer patients with low CCDC134 mRNA expression. $\mathrm{P}=2.0 \mathrm{e}-6)$.

A

Points
T stage
N stage
M stage
Age
PAM50
CCDC134
Total Points
Linear Predictor
1-year Survival Probability
3-year Survival Probability
5-year Survival Probability

T stage

$\mathrm{N}$ stage

$M$ stage

Age

PAM50

CCDC134

Total Points

5-year Survival Probability
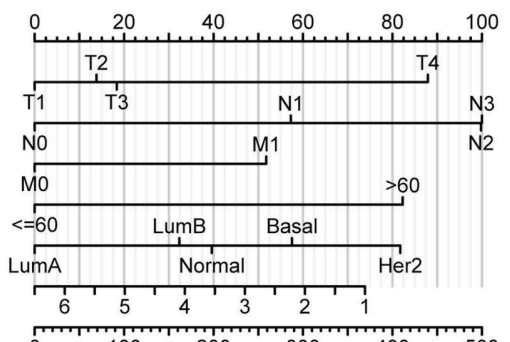

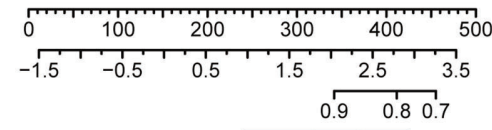

\begin{tabular}{lllll}
\cline { 2 - 3 } & 0.8 & 0.6 & 0.4 & 0.2 \\
0.8 & 0.6 & 0.4 & 0.2
\end{tabular}
B

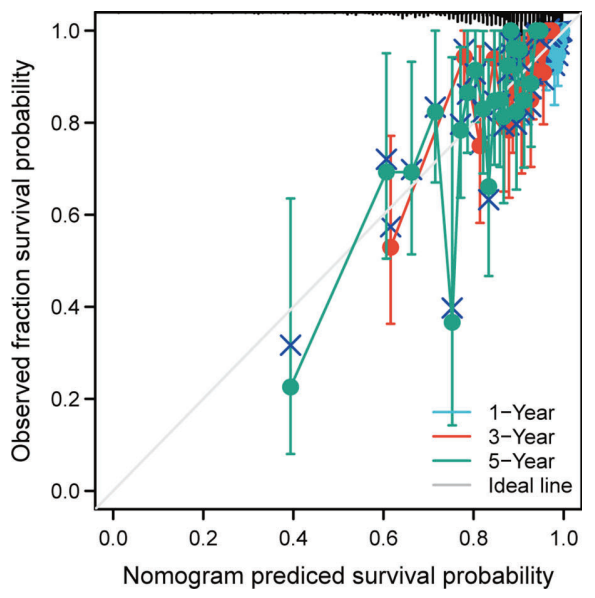

FIGURE 6 | Construction of a rosette to predict the probability of survival in BRCA patients. (A) Nomogram to predict 1 -, 3 -, and 5-year BRCA survival probabilities consisting of CCDC134 and independent clinical risk factors. (B) Nomograms calibrated to predict the probabilities of 1 -, 3 - and 5 -year survival. The gray line represents actual survival.

CD8 + T cells. This provides evidence that CCDC134 may be a potential member of the $\gamma$ c cytokine family (31).

Huang, J (32) showed that CCDC134 may act as a new regulator of hADA2a and play a role in the PCAF complex through hADA2a, affecting its acetyltransferase activity and UVinduced DNA damage repair. However, in breast cancer, CCDC134 expression and its prognostic value have not been fully studied. Systematic approaches to breast cancer include surgery, endocrine therapy, radiotherapy and targeted therapy, and Endocrine therapy is of great significance in the treatment of patients with hormone receptor positivity. In the estrogen receptor. Positive $(\mathrm{ER}+)$ patients, 5 years of adjuvant tamoxifen (that suppresses ER) reduces breast cancer mortality by approximately one third ( 0 - 14 years), recurrence about half ( 0 4 years) and about one third (5-9 years) (33). We found that the upregulation of CCDC134 was negatively correlated with hormones. In addition, since ER and PR status are correlated with prognosis, CCDC134 may be used as a predictor of hormone receptor status, and whether endocrine therapy biomark is needed. Furthermore, according to kaplan-Meier curves and log-rank tests, breast cancer patients with high mRNA expression had lower survival rates than those with low 


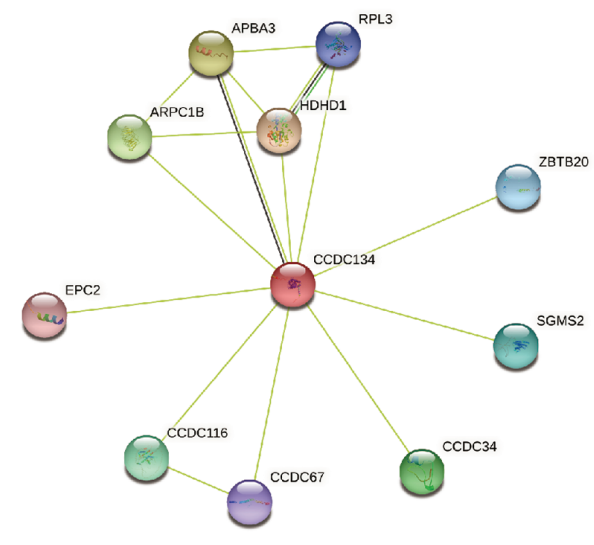

B

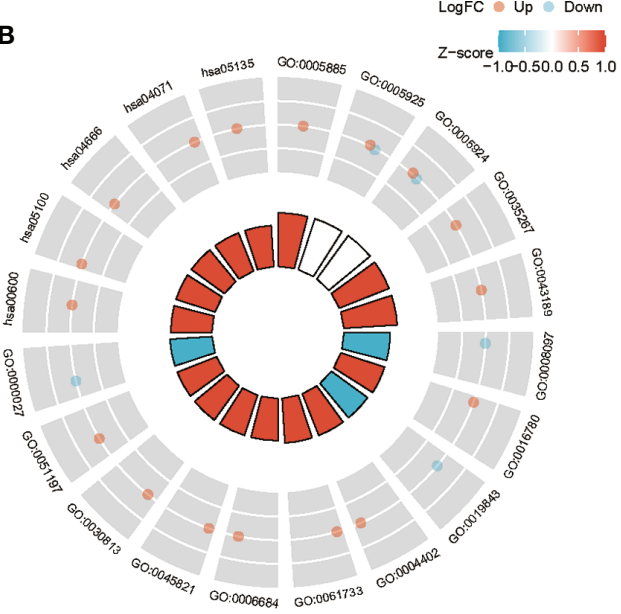

C
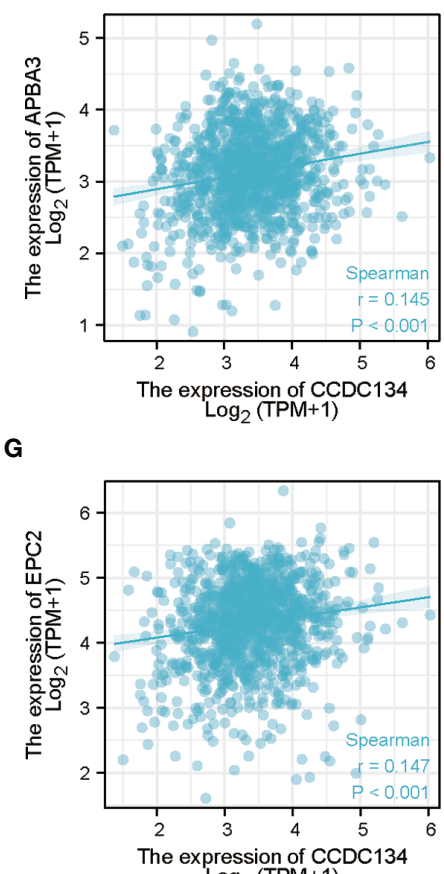

D

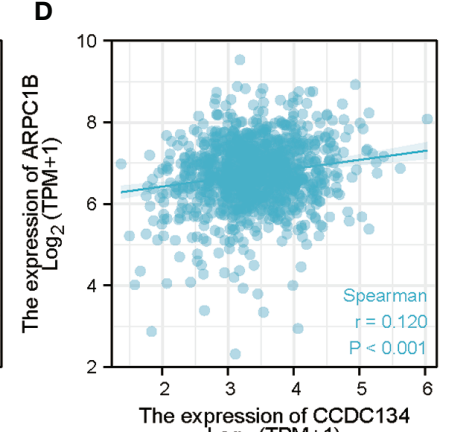

$\mathbf{H}$

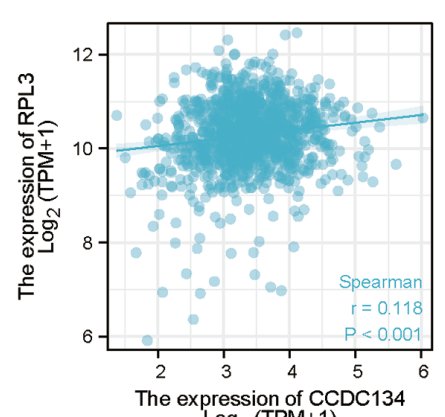

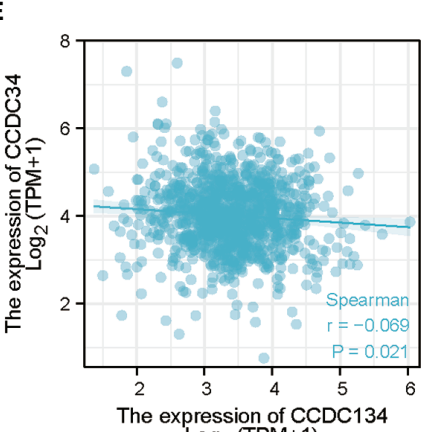

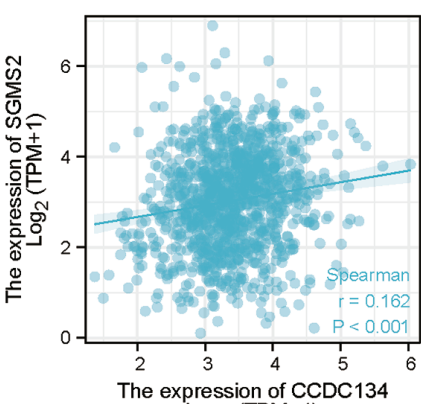

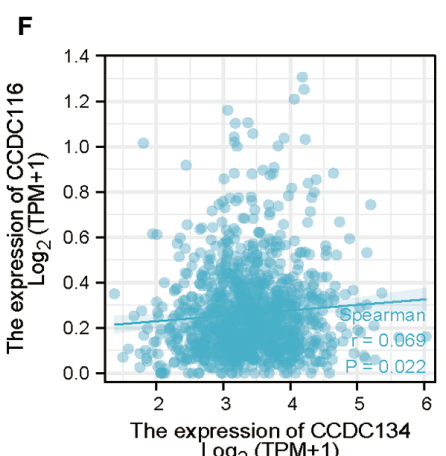

J

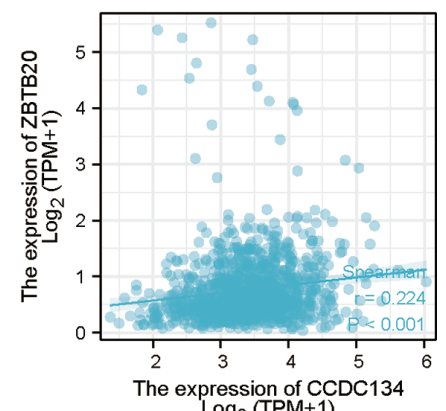

FIGURE 7 | PPI network and functional enrichment analysis. (A) CCDC134 and its co-expressed gene network. (B) Functional enrichment analysis of co-expressed genes. (C-J) The correlation analysis of CCDC134 expression and co-expressed genes. 


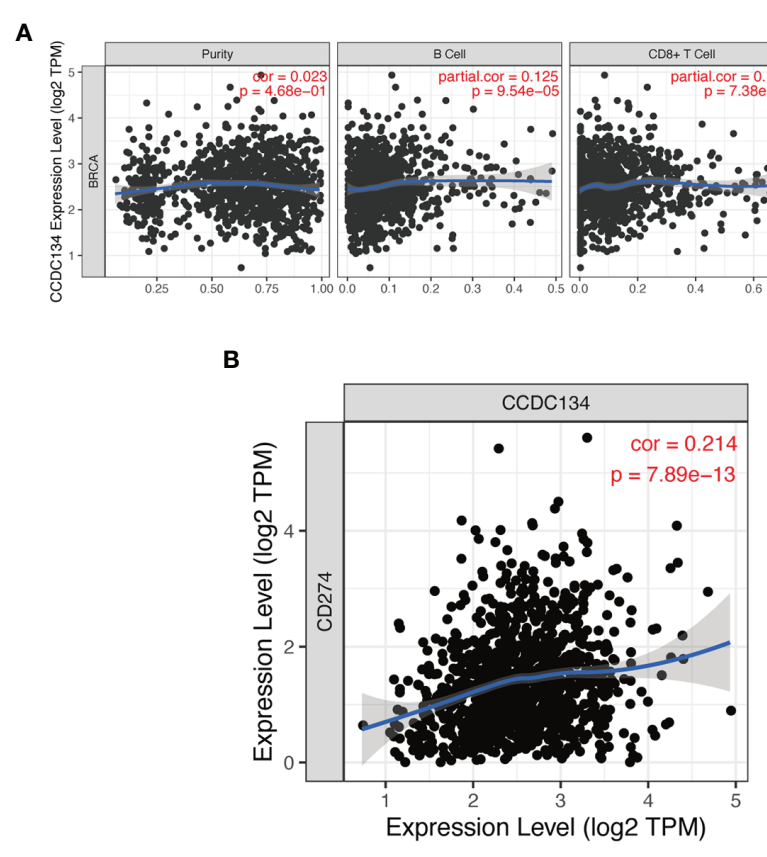

D

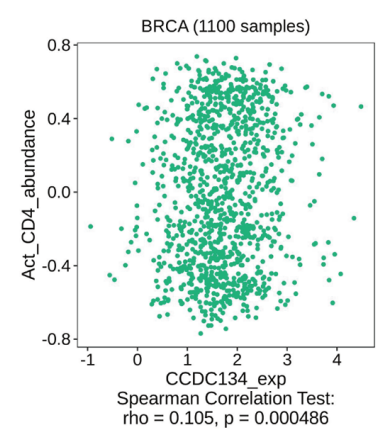

H

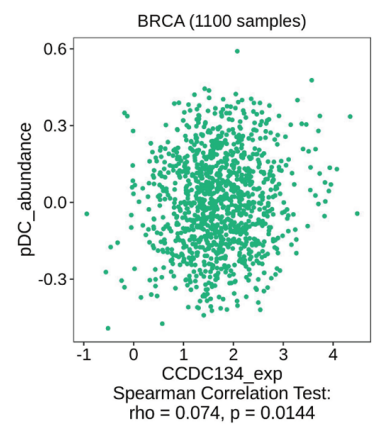

E

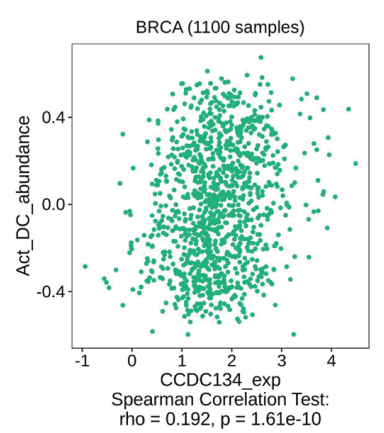

I

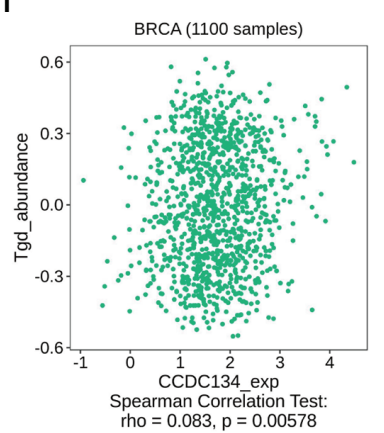

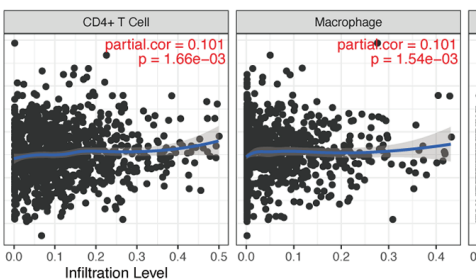
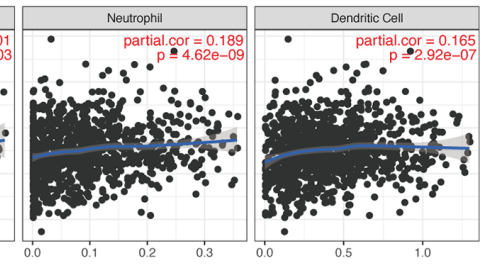

C

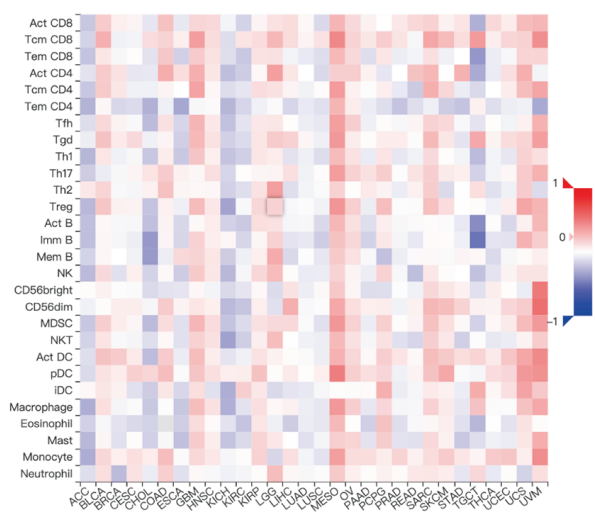

F

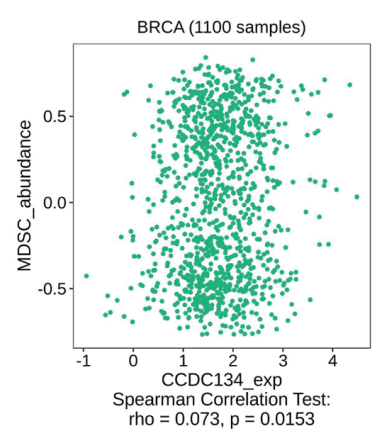

J

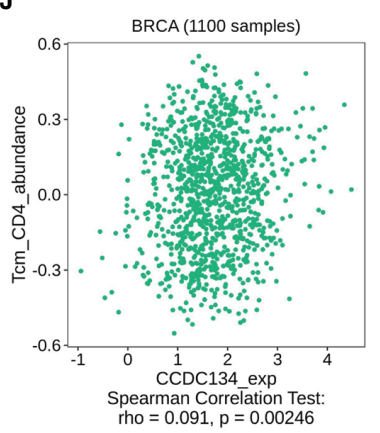

G

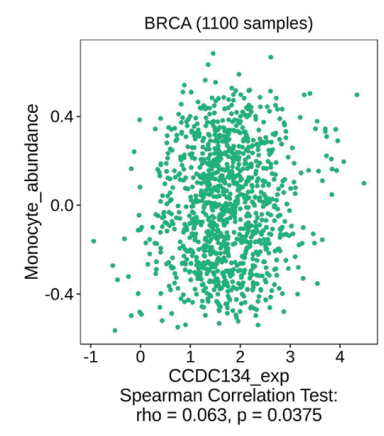

K

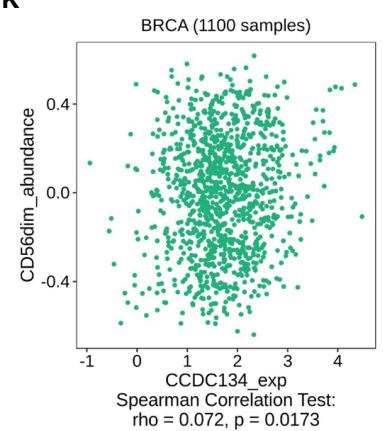

FIGURE 8 | CCDC134 expression and the immune level of correlation. (A) The expression of CCDC134 in breast cancer was correlated with tumor purity, B cells, CD4 + T cells, CD8 + T cells, macrophages, neutrophils and dendritic cells. (C) Relationship between CCDC134 expression and 28 lymphocyte species in human tumors. (B) CCDC134 expression was positively correlated with CD274(PD-L1) in breast cancer. (D-K) CCDC134 and CD4 + T cells ( $r=0.105, P=0.000486)$, DC cells $(r=0.192, P=1.61 E-10)$, MDSC cells $(r=0.073, P=0.0153)$, monocytes $(r=0.063, P=0.0375), p D C$ cells $(r=0.074, P=0.0144)$, Tgd cells $(r=0.083, P=$ 0.00578), Tcm_CD4 + T cells $(r=0.091, P=0.00246)$ and CD56DIM ( $r=0.072, P=0.0173)$. 
A

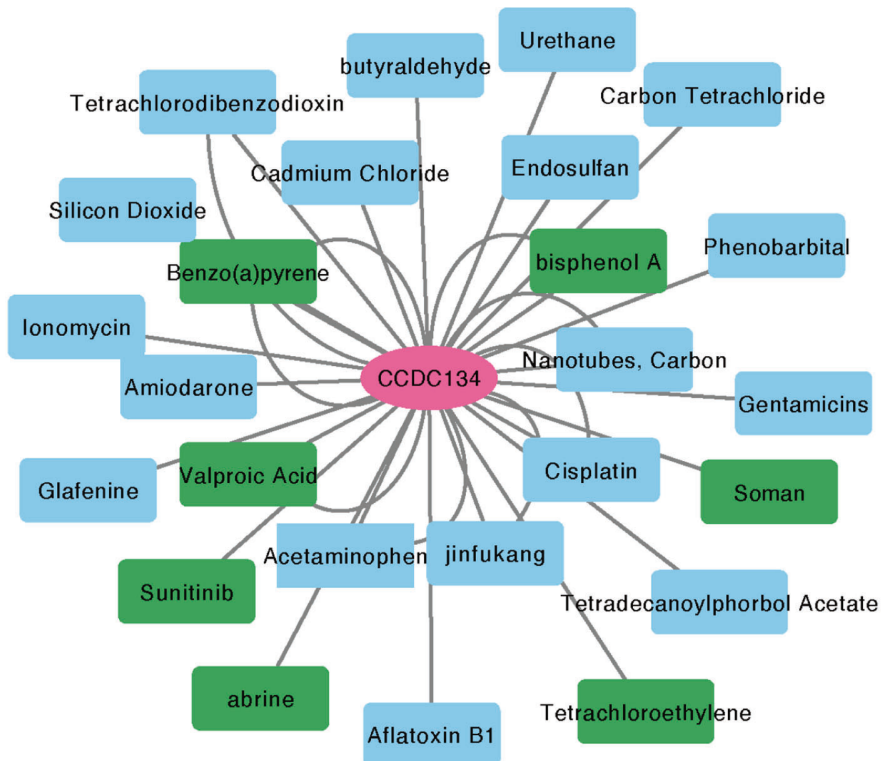

B

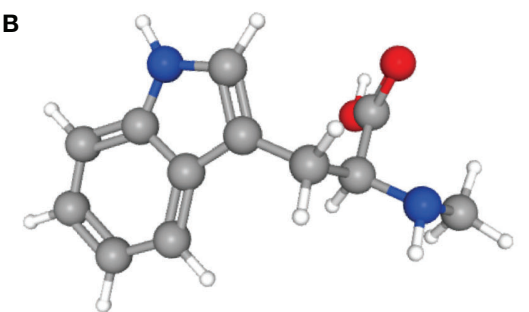

D

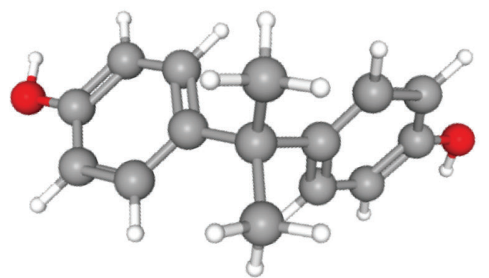

F

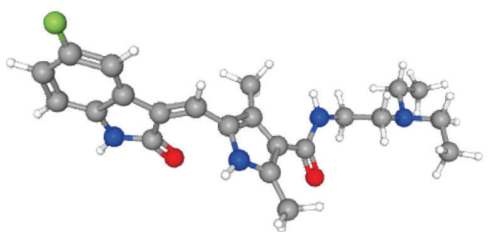

C

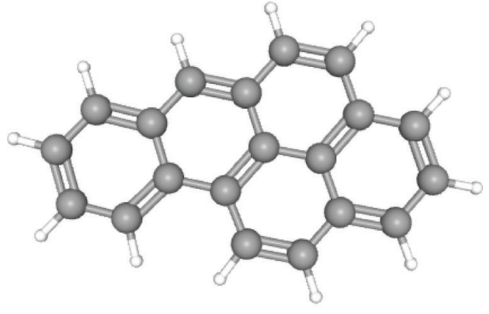

E

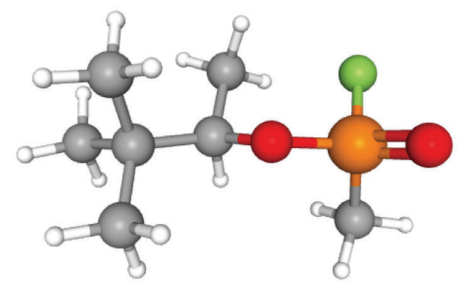

H

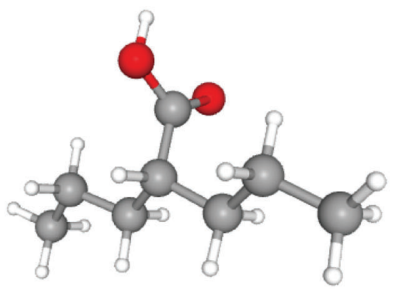

FIGURE 9 | Prediction of potential drug and molecular structure affecting CCDC134. (A) CTD database predicts potential drugs that affect CCDC134, with green representing drug molecules that inhibit CCDC134. (B-H) PubChem database predicts the molecular structures of seven targeted drugs. (B) abrine, (C) Benzo(A) Pyrene, (D) bisphenol A, (E) Soman, (F) Sunitinib, (G) Tetrachloroethylene, (H) Valproic Acid. 
CCDC134 levels. Based on our data, we conclude that CCDC134 can serve as a biomarker for poor prognosis in breast cancer. To identify patients with poor clinical prognosis.

There is little research on the possible role of CCDC134 in human lymphocytes. The correlation analysis between CCDC134 expression and immune cell infiltration in breast cancer has not been studied. In this study, we used TIMER to find that this gene could cause an increase in the number of infiltrating immune cells such as CD8+T in tumor tissues, but the expression of this gene was also positively correlated with the expression of PD-L1. Therefore, although this gene could recruit immune cells into tumor tissues, it could also lead to an increase in the expression of PD-L1 on the surface of tumor cells. Therefore, the high expression of this gene still has an inhibitory effect against tumor immune response. It is necessary to further study the mechanism and develop corresponding targeted drugs to remove the immunosuppressive effect of this gene and improve the survival prognosis of tumor patients.

By using the CTD database to predict drugs that inhibit CCDC134, seven drugs were identified. Previous studies supported abrine, Benzo(A) Pyrene, bisphenol A, Soman, Sunitinib, Tetrachloroethylene, and Valproic Acid can target CCDC134 in vitro and is expected to make new progress in the treatment of breast cancer. PubChem databases were used to determine the molecular structure of identified drugs.

There are several limitations to this study. Firstly, the expression and prognostic significance of CCDC134 were studied through online public database. Further studies on clinical samples and cell animal experiments are needed to verify these results. Secondly, in vitro and in vivo experiments need to be designed in order to further study the detailed mechanism of CCDC134 affecting immune invasion of breast cancer. Thirdly, besides CCDC134, there might be other novels genes associated with breast cancer (34). It might be better to study the association between them. Finally, all analyses in this study focus on bulk sequencing data. Fourthly, it might be better to study the role of CCDC134 using single cell sequencing to avoid cell heterogeneity using methods like single cell clustering (35-38).

In summary, in this study, we found for the first time that CCDC134 is highly up-regulated in breast cancer, and poor prognosis can be used as a potential prognostic marker and may play a specific role in immune infiltration.

\section{REFERENCES}

1. Sung H, Ferlay J, Siegel RL, Laversanne M, Soerjomataram I, Jemal A, et al. Global Cancer Statistics 2020: GLOBOCAN Estimates of Incidence and Mortality Worldwide for 36 Cancers in 185 Countries. CA Cancer J Clin (2021) 71:209-49. doi: 10.3322/caac.21660

2. Parkin DM, Bray F, Ferlay J, Pisani P. Global Cancer Statistic. CA Cancer J Clin (2005) 55:74-108. doi: 10.3322/canjclin.55.2.74

3. Jemal A, Bray F, Center MM, Ferlay J, Ward E, Forman D. Global Cancer Statistics. CA Cancer J Clin (2011) 61:69-90. doi: 10.3322/caac.20107

4. Torre LA, Bray F, Siegel RL, Ferlay J, Lortet-Tieulent J, Jemal A. Global Cancer Statistic. CA Cancer J Clin (2015) 65:87-108. doi: 10.3322/caac.21262

5. Bray F, Ferlay J, Soerjomataram I, Siegel RL, Torre LA, Jemal A. Global Cancer Statistics 2018: GLOBOCAN Estimates of Incidence and Mortality

\section{DATA AVAILABILITY STATEMENT}

The original contributions presented in the study are included in the article/Supplementary Material. Further inquiries can be directed to the corresponding authors.

\section{ETHICS STATEMENT}

Written informed consent was obtained from the individual(s) for the publication of any potentially identifiable images or data included in this article.

\section{AUTHOR CONTRIBUTIONS}

Authors HY and HW conceived and designed the study. ZH, LY, JC, and JH performed the experiments. YC, JC, SL, and JL analyzed the data. $\mathrm{ZH}$ wrote the manuscript. All authors have read and approved this manuscript.

\section{FUNDING}

This work was supported by The Natural Science foundation of Fujian province (No. 2020J011112) and Joint Funds for the innovation of science and Technology, Fujian province (Grant number: 2020Y9039).

\section{ACKNOWLEDGMENTS}

The authors thank reviewers for helpful comments on the manuscript.

\section{SUPPLEMENTARY MATERIAL}

The Supplementary Material for this article can be found online at: https://www.frontiersin.org/articles/10.3389/fonc.2022. 858487/full\#supplementary-material

Worldwide for 36 Cancers in 185 Countries. CA Cancer J Clin (2018) 68:394424. doi: $10.3322 /$ caac. 21492

6. Hortobagyi GN, de la Garza Salazar J, Pritchard K, Amadori D, Haidinger R, Hudis CA, et al. The Global Breast Cancer Burden: Variations in Epidemiology and Survival. Clin Breast Cancer (2005) 6:391-401. doi: 10.3816/CBC.2005.n.043

7. He B, Lang J, Wang B, Liu X, Lu Q, He J, et al. TOOme: A Novel Computational Framework to Infer Cancer Tissue-Of-Origin by Integrating Both Gene Mutation and Expression. Front Bioeng Biotechnol (2020) 8:394. doi: 10.3389/fbioe.2020.00394

8. Kanathezath A, Chembra V, Variyath SKP, Nair GG. Identification of Biomarkers and Functional Modules From Genomic Data in Stage-Wise Breast Cancer. Curr Bioinf (2021) 16:722-33. doi: 10.2174/1574893615999200 922123104 
9. Liu H, Qiu C, Wang B, Bing P, Tian G, Zhang X, et al. Evaluating DNA Methylation, Gene Expression, Somatic Mutation, and Their Combinations in Inferring Tumor Tissue-Of-Origin. Front Cell Dev Biol (2021) 9:619330. doi: $10.3389 /$ fcell.2021.619330

10. George K, Faziludeen S, Sankaran P, Joseph PK. Breast Cancer Detection From Biopsy Images Using Nucleus Guided Transfer Learning and Belief Based Fusion. Comput Biol Med (2020) 124:103954. doi: 10.1016/j.compbiomed.2020.103954

11. Rajarajan D, Kaur B, Penta D, Natesh J, Meeran SM. miR-145-5p as a Predictive Biomarker for Breast Cancer Stemness by Computational Clinical Investigation. Comput Biol Med (2021) 135:104601. doi: 10.1016/ j.compbiomed.2021.104601

12. Tian L, Wang SL. Exploring miRNA Sponge Networks of Breast Cancer by Combining miRNA-disease-lncRNA and miRNA-Target Networks. Curr Bioinf (2021) 16:385-94. doi: 10.2174/1574893615999200711171530

13. Huang J, Shi T, Ma T, Zhang Y, Ma X, Lu Y, et al. CCDC134, a Novel Secretory Protein, Inhibits Activation of ERK and JNK, But Not P38 MAPK. Cell Mol Life Sci (2008) 65:338-49. doi: 10.1007/s00018-007-7448-5

14. Huang J, Xiao L, Gong X, Shao W, Yin Y, Liao Q, et al. Cytokine-Like Molecule CCDC134 Contributes to CD8(+) T-Cell Effector Functions in Cancer Immunotherapy. Cancer Res (2014) 74:5734-45. doi: 10.1158/00085472.CAN-13-3132

15. Zhong J, Zhao M, Luo Q, Ma Y, Liu J, Wang J, et al. And Huang, CCCDC134 is Down-Regulated in Gastric Cancer and its Silencing Promotes Cell Migration and Invasion of GES-1 and AGS Cells via the MAPK Pathway. Mol Cell Biochem (2013) 372:1-8. doi: 10.1007/s11010-012-1418-4

16. Tomczak K, Czerwinska P, Wiznerowicz M. The Cancer Genome Atlas (TCGA): An Immeasurable Source of Knowledge. Contemp Oncol (Pozn) (2015) 19:A68-77. doi: 10.5114/wo.2014.47136

17. Edwards NJ, Oberti M, Thangudu RR, Cai S, Mcgarvey PB, Jacob S, et al. The CPTAC Data Portal: A Resource for Cancer Proteomics Research. J Proteome Res (2015) 14:2707-13. doi: 10.1021/pr501254j

18. Chandrashekar DS, Bashel B, Balasubramanya SAH, Creighton CJ, PonceRodriguez I, Chakravarthi B, et al. UALCAN: A Portal for Facilitating Tumor Subgroup Gene Expression and Survival Analyses. Neoplasia (2017) 19:64958. doi: 10.1016/j.neo.2017.05.002

19. Uhlen M, Fagerberg L, Hallstrom BM, Lindskog C, Oksvold P, Mardinoglu A, et al. Proteomics. Tissue-Based Map of the Human Proteome. Science (2015) 347:1260419. doi: 10.1126/science.1260419

20. Yu G, Wang LG, Han Y, He QY. Clusterprofiler: An R Package for Comparing Biological Themes Among Gene Clusters. OMICS (2012) 16:284-7. doi: 10.1089/omi.2011.0118

21. Liu J, Lichtenberg T, Hoadley KA, Poisson LM, Lazar AJ, Cherniack AD, et al. An Integrated TCGA Pan-Cancer Clinical Data Resource to Drive HighQuality Survival Outcome Analytics. Cell (2018) 173:400-16.e411. doi: 10.1016/j.cell.2018.02.052

22. Szklarczyk D, Franceschini A, Kuhn M, Simonovic M, Roth A, Minguez P, et al. The STRING Database in 2011: Functional Interaction Networks of Proteins, Globally Integrated and Scored. Nucleic Acids Res (2011) 39:D561-8. doi: $10.1093 / \mathrm{nar} / \mathrm{gkq} 973$

23. Bashiri H, Rahmani H, Bashiri V, Modos D, Bender A. EMDIP: An Entropy Measure to Discover Important Proteins in PPI Networks. Comput Biol Med (2020) 120:103740. doi: 10.1016/j.compbiomed.2020.103740

24. Wu C, Lin B, Shi K, Zhang Q, Gao R, Yu Z, et al. PEPRF: Identification of Essential Proteins by Integrating Topological Features of PPI Network and Sequence-Based Features. via Random Forest Curr Bioinf (2021) 16:1161-8. doi: 10.2174/1574893616666210617162258

25. Li T, Fan J, Wang B, Traugh N, Chen Q, Liu JS, et al. TIMER: A Web Server for Comprehensive Analysis of Tumor-Infiltrating Immune Cells. Cancer Res (2017) 77:e108-10. doi: 10.1158/0008-5472.CAN-17-0307

26. Ru B, Wong CN, Tong Y, Zhong JY, Zhong SSW, Wu WC, et al. TISIDB: An Integrated Repository Portal for Tumor-Immune System Interactions. Bioinformatics (2019) 35:4200-2. doi: 10.1093/bioinformatics/btz210
27. Robin X, Turck N, Hainard A, Tiberti N, Lisacek F, Sanchez JC, et al. pROC: An Open-Source Package for R and S+ to Analyze and Compare ROC Curves. BMC Bioinf (2011) 12:77. doi: 10.1186/1471-2105-12-77

28. Chen L, Li J, Chang M. Cancer Diagnosis and Disease Gene Identification via Statistical Machine Learning. Curr Bioinf (2020) 15:956-62. doi: 10.2174/ 1574893615666200207094947

29. Zhang Z, Chai H, Wang Y, Pan Z, Yang Y. Cancer Survival Prognosis With Deep Bayesian Perturbation Cox Network. Comput Biol Med (2021) 141:105012-2. doi: 10.1016/j.compbiomed.2021.105012

30. Dubail J, Brunelle P, Baujat G, Huber C, Doyard M, Michot C, et al. Homozygous Loss-Of-Function Mutations in CCDC134 Are Responsible for a Severe Form of Osteogenesis Imperfecta. J Bone Miner Res (2020) 35:1470-80. doi: 10.1002/jbmr.4011

31. Yu B, Zhang T, Xia P, Gong X, Qiu X, Huang J. CCDC134 Serves a Crucial Role in Embryonic Development. Int J Mol Med (2018) 41:381-90. doi: 10.3892/ijmm.2017.3196

32. Huang J, Zhang L, Liu W, Liao Q, Shi T, Xiao L, et al. CCDC134 Interacts With Hada2a and Functions as a Regulator of Hada2a in Acetyltransferase Activity, DNA Damage-Induced Apoptosis and Cell Cycle Arrest. Histochem Cell Biol (2012) 138:41-55. doi: 10.1007/s00418-012-0932-5

33. Early Breast Cancer Trialists' Collaborative, G, Davies C, Godwin J, Gray R, Clarke M, Cutter D, et al. Relevance of Breast Cancer Hormone Receptors and Other Factors to the Efficacy of Adjuvant Tamoxifen: Patient-Level MetaAnalysis of Randomised Trials. Lancet (2011) 378:771-84. doi: 10.1016/ S0140-6736(11)60993-8

34. Zhang Y, Xiang J, Tang L, Li J, Lu Q, Tian G, et al. Identifying Breast CancerRelated Genes Based on a Novel Computational Framework Involving KEGG Pathways and PPI Network Modularity. Front Genet (2021) 12:596794. doi: 10.3389/fgene.2021.596794

35. Xu J, Cai L, Liao B, Zhu W, Yang J. CMF-Impute: An Accurate Imputation Tool for Single-Cell RNA-Seq Data. Bioinformatics (2020) 36:3139-47. doi: 10.1093/bioinformatics/btaa109

36. Hasan MZ, Islam S, Matsumoto K, Kawai T. Meta-Analysis of Single-Cell RNA-Seq Data Reveals Phenotypic Switching of Immune Cells in Severe COVID-19 Patients. Comput Biol Med (2021) 137:104792. doi: 10.1016/ j.compbiomed.2021.104792

37. Turki T, Taguchi YH. Discriminating the Single-Cell Gene Regulatory Networks of Human Pancreatic Islets: A Novel Deep Learning Application. Comput Biol Med (2021) 132:104257. doi: 10.1016/j.compbiomed. 2021.104257

38. Zhuang J, Cui L, Qu T, Ren C, Yang J. A Streamlined scRNA-Seq Data Analysis Framework Based on Improved Sparse Subspace Clustering. IEEE Access (2021) 9:9719-27. doi: 10.1109/ACCESS.2021.3049807

Conflict of Interest: The authors declare that the research was conducted in the absence of any commercial or financial relationships that could be construed as a potential conflict of interest.

Publisher's Note: All claims expressed in this article are solely those of the authors and do not necessarily represent those of their affiliated organizations, or those of the publisher, the editors and the reviewers. Any product that may be evaluated in this article, or claim that may be made by its manufacturer, is not guaranteed or endorsed by the publisher.

Copyright $\odot 2022$ Huang, Yang, Chen, Li, Huang, Chen, Liu, Wang and Yu. This is an open-access article distributed under the terms of the Creative Commons Attribution License (CC BY). The use, distribution or reproduction in other forums is permitted, provided the original author(s) and the copyright owner(s) are credited and that the original publication in this journal is cited, in accordance with accepted academic practice. No use, distribution or reproduction is permitted which does not comply with these terms. 